\title{
Increasing the Reliability of Applications in AAL by Distinguishing Moving Persons from Pets by Means of a Sensor Floor
}

\author{
Raoul Hoffmann, Dr. Axel Steinhage, Christl Lauterbach \\ Future-Shape GmbH, Altlaufstr. 34, 85635 Höhenkirchen-Siegertsbrunn, Germany \\ raoul.hoffmann@future-shape.com
}

\begin{abstract}
:
We introduce a method to track movement in indoor environments and distinguish persons from pets. The system uses sensor data from SensFloor ${ }^{\circledR}$, a capacitive sensor that is installed in large areas under the floor and which is capable of detecting the presence and location of persons and animals moving on it. Our algorithm determines the location of entities on the floor in a first step, and then further analyses the location changes for typical movement patterns caused by humans or small animals. Tests with cats and persons walking over a SensFloor installation showed that our algorithm can reliably separate the cat and person activation patterns. We propose that applications in the area of Ambient Assisted Living (AAL) and Smart Homes that depend on information about the locations of persons, can benefit from the additional information if a tracked object is a person or a pet.
\end{abstract}

Key words: capacitive sensor, tracking, presence detection, smart homes, ambient assisted living

\section{Introduction}

Many applications in AAL and Smart Homes demand a high amount of detailed information about what is going on inside or around the house, to be able to react intelligently and in a useful manner. This typically includes information about the state of the house itself, like which devices like lights, air conditioning or the stove are switched on or off, if doors are locked etc., but also covers knowledge about the inhabitants, as: Is there currently anyone in the house, and if, how many and where exactly are they?

A typical exemplary application for which one needs to know about locations of persons in a house is to intelligently control lights in a building. In this case, the desired behaviour is to switch on the light in rooms where someone is currently in, and to switch off the lights in those where nobody is. The detection of the presence of persons in rooms can be implemented by using PIR-based (passive infrared) motion detectors [7]. These sensors detect infrared radiation that is emitted from persons, and react if such a radiation source moves inside their field of view. A common problem with this approach is that PIR-based motion sensors only react on moving objects, therefore if a person enters a room, the light will be switched as intended, but if the person then

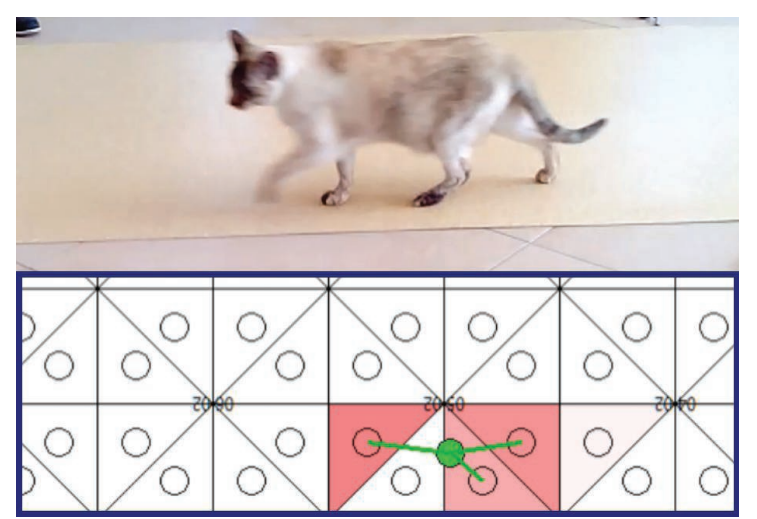

Fig. 1: Typical SensFloor activation pattern of a cat. The triangles are the sensor shapes, with a circle inside at the centroid. Darker red means higher capacitance. The green circle shows the estimated object position.

stops moving, for example sitting down at a desk, the lights will eventually be switched off again, provoking the person to frantically wave their hands in the air to trigger the sensor again.

Another property of PIR-based motion sensors is that they do not deliver a position estimate, but only binary information about something moving into a defined field of view, which is also subject to occlusion by obstacles inside the line of sight. An additional issue is that PIR-based motion sensors are not only triggered by humans, but also animals. This can result in unintended behaviour of an automation 

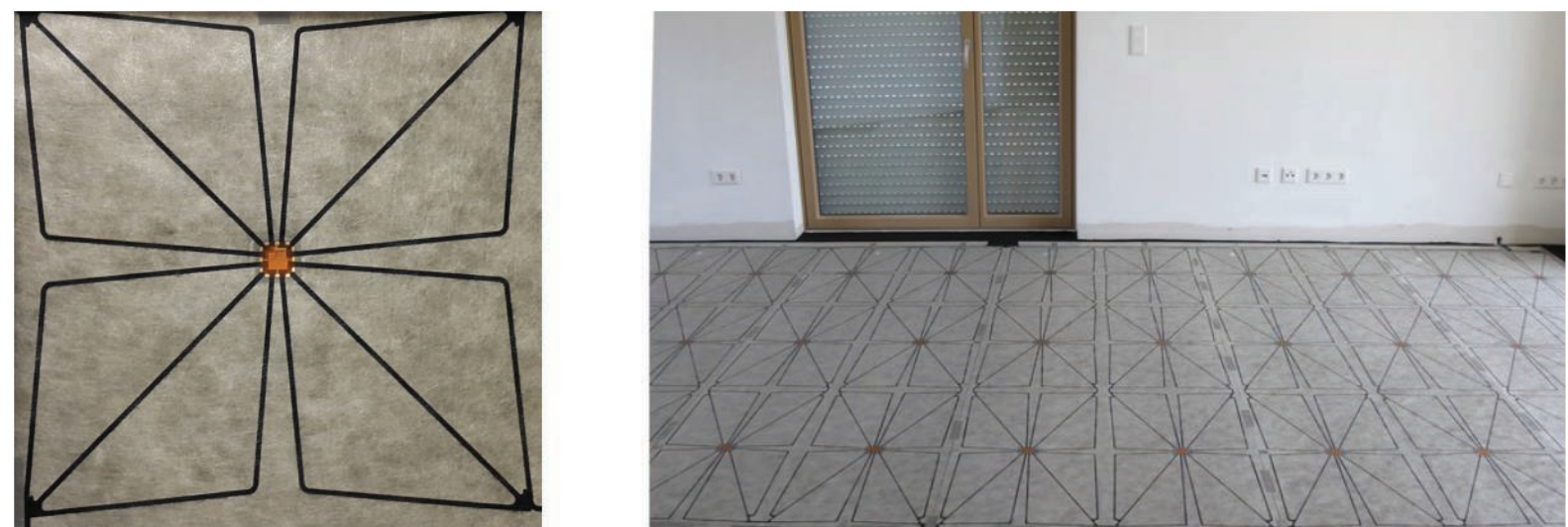

Fig. 2: Left side: SensFloor Module. The grey textile is the conductive coating, at the black lines the conductive layer was removed such that the black base layer of polyester fleece shines through. The embedded electronic module can be seen in the middle as a small orange rectangle. Right side: Typical large-area installation of SensFloor in a living room.

application, resulting in decreased acceptance and annoyance of the inhabitants.

In this work, we address these issues by presenting a system that is capable of delivering precise localizations of persons in a room, and which can distinguish persons from certain pets like cats.

\section{SensFloor Capacitive Sensor System}

SensFloor [1],[2] consists of electronic modules embedded in a composite textile in a regular grid. The system provides electric capacitance measurements in spatially discretised defined areas on the floor.

The textile base is a fleece underlay with a thickness of $3 \mathrm{~mm}$ as typically used as an impact sound absorption layer in floor building. Subsequently, the base is coated with a thin layer of a conductive fleece. Into the conductive layer, different shapes can be carved by CNCcutting and then partly removing stripes of the material. By this, areas that are electrically connected can be separated from each other.

This process is applied to create a shape that consists of the defined areas used as capacitor plates for the capacitive measurement as well as traces used as power supply lines to the embedded electronics. This shape is shown in Fig. 2.

The eight triangle-shaped areas as seen in this figure are used for the capacitance measurement, while the lines going away from the electronic modules deliver the supply voltage.

The electronic measurement modules are embedded into the composite textile by a bonding process. Each module is a circuit board of a flexible material which withstands a certain amount of mechanical deformation. The electronics consist of voltage regulation and a micro-controller that carries out the electric capacitance measurements on the sensor areas. The sensor readings are transmitted via $868 \mathrm{Mhz}$ wireless radio.

The single modules operate independently from each other, and only send out messages whenever a capacitance value changes. The 16-byte message contains a unique address id to assign the message to a module on the floor, and the capacitance values of the eight measurement areas.

The electric capacitance on the floor is changed by persons and animals walking over the floor. A capacitance change also happens when other conductive materials are on the floor, like water and other liquids, or objects made out of metal.

It is emphasized that SensFloor reacts on the proximity of conductive materials, not the mechanical pressure. Therefore, it can be installed under virtually any floor covering that is non-conducting.

A receiver unit receives the messages that are transmitted wirelessly from the sensor modules embedded in the floor, and forwards them via USB to a PC for further processing.

\section{Tracking and Classifying cats and humans}

In software the signals from the SensFloor receiver are provided on a virtual serial port as a stream of data messages that consist of the capacitance changes that happen on the floor, together with the respective id of the module that sent the message. A lookup-table of ids provides the world positions of modules in the 

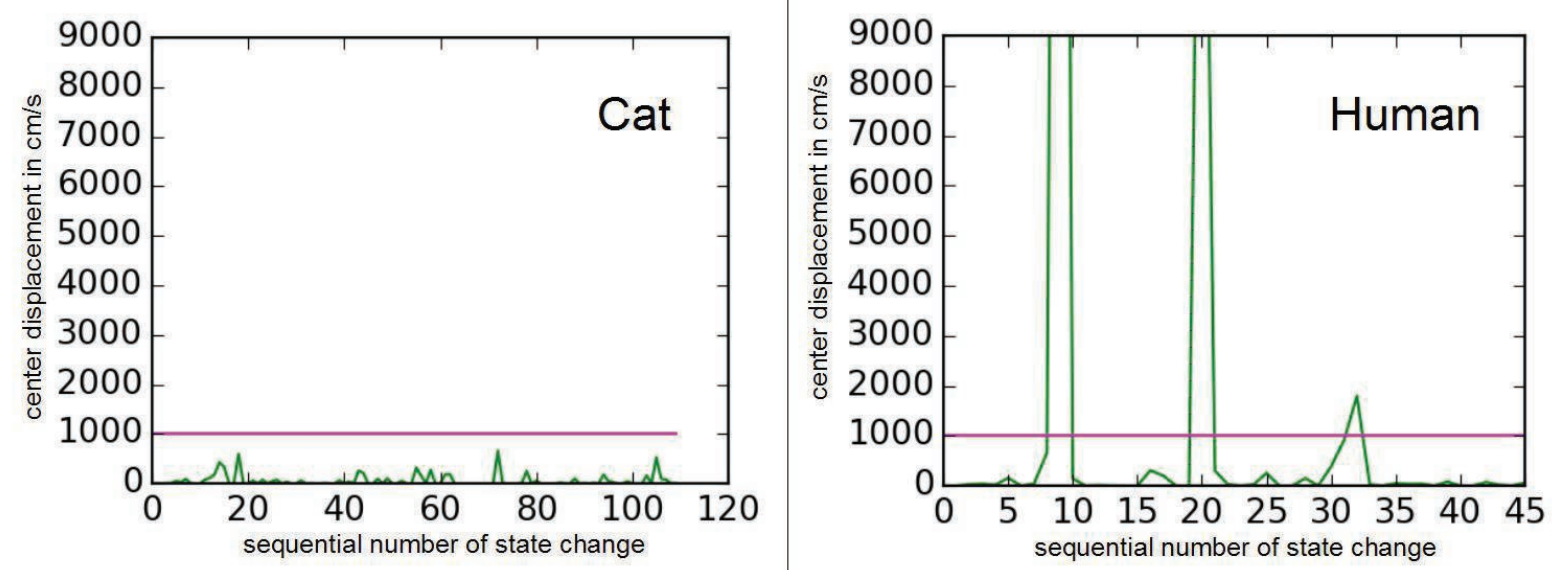

corresponding track:

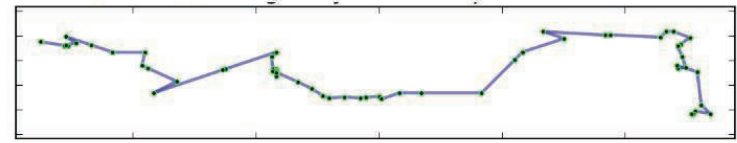

corresponding track:

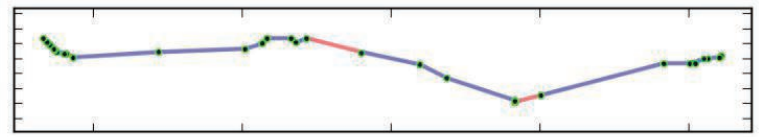

Fig. 3: These graphs show tracked object velocities between two consecutive timesteps as determined from a cat and a human walking over the floor. The magenta-coloured line denotes the threshold of $1000 \mathrm{~cm} / \mathrm{s}$. Each time a person takes a step, the calculated location experiences an abrupt change. Below, the corresponding $2 D$ track is plotted, translation segments that exceed the $1000 \mathrm{~cm} / \mathrm{s}$ threshold are coloured in red, the others in blue.

room. Taking the modules world position and orientation together with the known shape of the single sensor areas connected to the module allows the calculation of the area centroid.

A geometric map of the current capacitance is updated for each message. This map holds the current state of the electric capacitance distribution in the room, stored as a list of sensor field centroid coordinates together with the measured capacitance. In our setup, object locations were tracked by calculating the center-of-gravity from all observed capacities in a time step.

The location $\vec{p}_{o}$ of an observed object is calculated by a center-of-gravity-like summation of all $n$ map entries consisting of the sensor field centroid $\vec{p}_{s, i}$ and a corresponding sensor activation $c_{i}$ :

$$
\vec{p}_{o}=\frac{1}{C} \sum_{i=1}^{n} c_{i} \vec{p}_{s, i} \text { where } C=\sum_{i=1}^{n} c_{i}
$$

Cats and humans are very different in anatomy. The physical properties that differ the most are the two more legs that cats have, the shorter leg length, and different gait cycle, resulting in a shorter step length. [3], [4], [5], [6]

These differences constrain the way humans and cats move respectively, and become noticeable in the sensor data. A typical activation pattern is shown in fig. 1 .

The movement of the calculated position of a human over time appears to be more jerky, with sudden translations of the object centre visible for every step he takes. In contrast, cats move very smoothly resulting in a calculated trajectory with no sudden jumps of the calculated position.

The classification algorithm takes the localisation updates of the trajectory (1) as input, and finds these sudden translations of the position. This happens by discrete derivation of the translation according to the time $t$ for each update at timestep $j$, resulting in the signal velocity $s_{j}$ of the object:

$$
\vec{s}_{j}=\frac{\vec{p}_{o, j}-\vec{p}_{o, j-1}}{t_{j}-t_{j-1}}
$$

A moving object is classified as a human if this velocity $s_{j}$ exceeds a certain threshold, e.g. $10.0 \mathrm{~m} / \mathrm{s}$.

Note that this velocity of the calculated object location for a single time step does not correspond to the physical velocity of the object. However, the physical velocity can be determined by averaging the object velocities over several timesteps. 

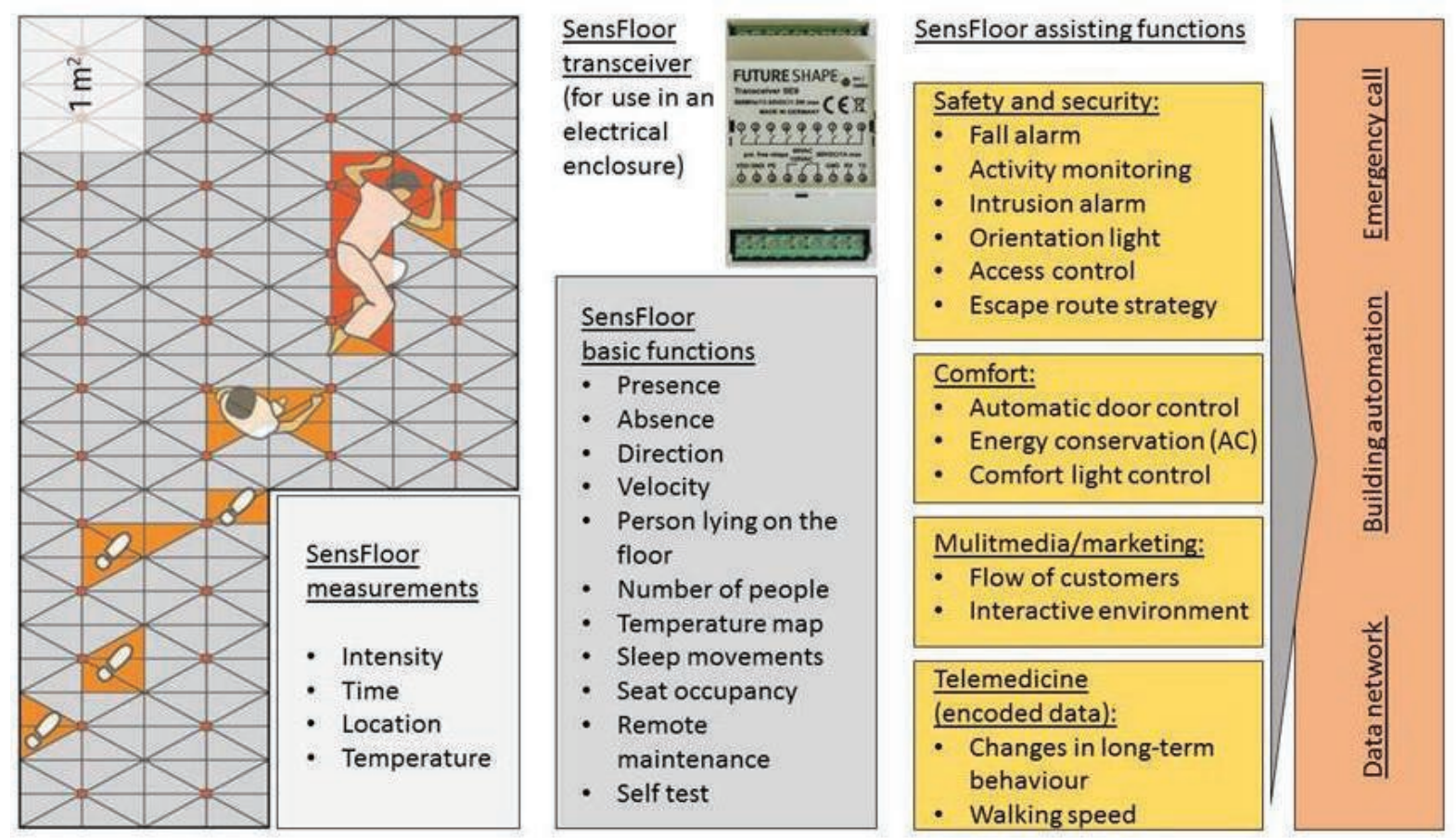

Fig. 4: Typical Applications of SensFloor. Radio messages from the floor contain the direct measurements the module is capable of, in particular the electric capacitance. These are transmitted to a SensFloor transceiver which collects and analyses information from many modules. Higher-level applications are listed on the right side.

\section{Experimental Results}

We tested our method with data captured on a patch of SensFloor sized $1 \mathrm{~m} \times 3.5 \mathrm{~m}$. The sensor area was filmed during the experiment for later manual assignment of trajectory data to the two classes "cats" and "humans". This manual labelling was used as ground truth against which the algorithm was compared.

A cat was set free and the SensFloor data generated by her movement recorded by a datalogger. The experiment was then repeated with persons walking on the sensor area.

The recorded datasets were manually labelled according to the corresponding video proof of the experiments.

The algorithm succeeded to distinguish the cat datasets from the human datasets with an accuracy of $100 \%$. As a further result, the algorithm counted the numbers of steps in the human datasets with a typical error of $+-8 \%$.

\section{Applications \& Impact}

The SensFloor system and applications where it was used successfully already are shown in fig. 4.

We see a main field of application of our new method in home automation tasks whenever it is mandatory to reliably know about peoples locations and avoid false alarms caused by pets.

For example, with this system doors can be controlled to open automatically, but only for humans. One can imagine a setup where pets live in shared space with humans and move freely, but where some rooms like e.g. the kitchen are restricted for hygienic reasons and not accessible for pets.

In particular for AAL applications, which are often targeted at elderly people, the possibility to use supporting technology that is robust against interference from animals can prove exceptionally useful. Studies like [8] show that caring for a pet can improve mental and physical health. This suggests that elderly people who are pet owners and require assistance should be allowed to keep their pets and not be forced to give them away due to technological restrictions.

\section{Restrictions \& Outlook}

The system as it is currently implemented and as it was used for our experiments requires that the floor area is occupied by a single entity at a time, as the object position is calculated as the center-of-gravity of sensor activations over the whole floor area. Consequently, it is not yet 
suitable for deployment, as it proves to be deliberately useful in cases where areas are shared by persons and pets simultaneously.

Currently, we pursue research to combine the cat classification algorithm with an algorithm that is capable of clustering the SensFloor sensor data, therefore providing a separation of entities in advance. The cat classification algorithm can possibly be applied to these preseparated sensor activation clusters. We expect that this combination results in a tracking and classification method suitable for areas that are concurrently shared by multiple pets and persons.

The current implementation only separates cats from humans, though there are more pets to consider. We currently investigate to generalise the approach to include dogs in the classification.

\section{References}

[1] Lauterbach, C., Steinhage, A., Techmer, A.: "A Large-Area Sensor System Underneath the Floor for Ambient Assisted Living Applications", 2013, Pervasive and Mobile Sensing and Computing for Healthcare, Springer, 69-87

[2] Steinhage, A., Lauterbach, C.: "SensFloor and NaviFloor: Large-Area Sensor Systems Beneath your Feet", 2011 Nak-Young Chong, Fulvio Mastrogiovanni (eds.) Handbook of Research on Ambient Intelligence and Smart Environments: Trends and Perspectives, IGI Global Hershey, PA (USA)

[3] Lacquaniti, F., Grasso, R., Zago, M.: "Motor patterns in walking", 1999, Physiology 14(4), 168174

[4] Miller, S., Van Der Burg, J., Van der Meche, F. G. A.: "Locomotion in the cat: basic programmes of movement.", 1975, Brain research 91(2): 239253

[5] English, A. W., Lennard, P. R.: "Interlimb coordination during stepping in the cat: in-phase stepping and gait transitions", 1982, Brain Research 245(2), 353-364

[6] Janusz Blaszczyk, G. E. L.: "Why cats pace on the treadmill", 1993 Physiology \& Behavior 3, 501-507

[7] Zhang, Z., Gao, X., Biswas, J., Wu, J. K.: "Moving targets detection and localization in passive infrared sensor networks", 2007, 10th International Conference on Information Fusion, 1-6.

\section{Summary}

We presented a method to determine the location of objects by analysing data from SensFloor, a capacitive sensor floor hidden below common floor covering. The algorithm can classify detected objects between humans and cats by inspecting the differences in the signal patterns caused by the different walking gaits. Our method is useful in robust implementations of $A A L$ and home automation tasks, when it is important to track persons and avoid false alarms caused by pets.

No cats were harmed during the research and experiments for this work. Special thanks goes to Miguel Sousa for recording the first batch of data from walking cats.

[8] Serpell, J.: "Beneficial Effects of Pet Ownership on Some Aspects of Human Health and Behaviour", 1991, Journal of the Royal Society of Medicine 84(12), 717-720 